\title{
Histopathological features predictive of local control of atypical meningioma after surgery and adjuvant radiotherapy
}

\author{
William C. Chen, BS, ${ }^{1}$ Stephen T. Magill, MD, PhD, ${ }^{2}$ Ashley Wu, BS, ${ }^{1}$ \\ Harish N. Vasudevan, MD, PhD, ${ }^{1}$ Olivier Morin, PhD, ${ }^{1}$ Manish K. Aghi, MD, PhD, ${ }^{2}$ \\ Philip V. Theodosopoulos, MD, ${ }^{2}$ Arie Perry, MD, ${ }^{2,3}$ Michael W. McDermott, MD, ${ }^{2}$ \\ Penny K. Sneed, MD, ${ }^{1}$ Steve E. Braunstein, MD, PhD, ${ }^{1}$ and David R. Raleigh, MD, PhD' \\ Departments of ${ }^{1}$ Radiation Oncology, ${ }^{2}$ Neurological Surgery, and ${ }^{3}$ Pathology, University of California, San Francisco, California
}

\begin{abstract}
OBJECTIVE The goal of this study was to investigate the impact of adjuvant radiotherapy (RT) on local recurrence and overall survival in patients undergoing primary resection of atypical meningioma, and to identify predictive factors to inform patient selection for adjuvant RT.
\end{abstract}

METHODS One hundred eighty-two patients who underwent primary resection of atypical meningioma at a single institution between 1993 and 2014 were retrospectively identified. Patient, meningioma, and treatment data were extracted from the medical record and compared using the Kaplan-Meier method, log-rank tests, multivariate analysis (MVA) Cox proportional hazards models with relative risk (RR), and recursive partitioning analysis.

RESULTS The median patient age and imaging follow-up were 57 years (interquartile range [IQR] 45-67 years) and 4.4 years (IQR 1.8-7.5 years), respectively. Gross-total resection (GTR) was achieved in 114 cases (63\%), and 42 patients $(23 \%)$ received adjuvant RT. On MVA, prognostic factors for death from any cause included GTR (RR $0.4,95 \% \mathrm{Cl}$ $0.1-0.9, p=0.02$ ) and MIB1 labeling index (LI) $\leq 7 \%$ (RR 0.4, 95\% Cl 0.1-0.9, p = 0.04). Prognostic factors on MVA for local progression included GTR (RR $0.2,95 \% \mathrm{Cl} 0.1-0.5, \mathrm{p}=0.002)$, adjuvant $\mathrm{RT}(\mathrm{RR} 0.2,95 \% \mathrm{Cl} 0.1-0.4, \mathrm{p}<0.001)$, MIB1 LI $\leq 7 \%$ (RR 0.2, 95\% Cl 0.1-0.5, p < 0.001), and a remote history of prior cranial RT (RR 5.7, 95\% Cl 1.3-18.8, p $=0.03$ ). After GTR, adjuvant RT (0 of 10 meningiomas recurred, $p=0.01)$ and $\mathrm{MIB} 1 \mathrm{LI} \leq 7 \%(\mathrm{RR} 0.1,95 \% \mathrm{Cl} 0.003-0.3$, $\mathrm{p}<0.001$ ) were predictive for local progression on MVA. After GTR, $2.2 \%$ of meningiomas with $\mathrm{MIB} 1 \mathrm{LI} \leq 7 \%$ recurred (1 of 45), compared with $38 \%$ with MIB1 LI > 7\% (13 of 34; $p<0.001$ ). Recursive partitioning analysis confirmed the existence of a cohort of patients at high risk of local progression after GTR without adjuvant RT, with MIB1 LI > 7\%, and evidence of brain or bone invasion. After subtotal resection, adjuvant RT (RR 0.2, 95\% Cl 0.04-0.7, $p=0.009)$ and $\leq 5$ mitoses per $10 \mathrm{hpf}(\mathrm{RR} 0.1,95 \% \mathrm{Cl} 0.03-0.4, \mathrm{p}=0.002)$ were predictive on MVA for local progression.

CONCLUSIONS Adjuvant RT improves local control of atypical meningioma irrespective of extent of resection. Although independent validation is required, the authors' results suggest that MIB1 LI, the number of mitoses per 10 hpf, and brain or bone invasion may be useful guides to the selection of patients who are most likely to benefit from adjuvant RT after resection of atypical meningioma.

https://thejns.org/doi/abs/10.3171/2017.9.JNS171609

KEYWORDS atypical meningioma; MIB1; radiation; radiotherapy; oncology

$\mathrm{M}$ ENINGIOMAS are the most common primary CNS neoplasm, and comprise $20 \%-35 \%$ of all primary brain tumors. ${ }^{9,28}$ Although most meningiomas are effectively treated with surgery, a subset follows an aggressive clinical course characterized by local recurrence and poor survival. ${ }^{8,26}$ Atypical meningiomas (WHO grade II) display intermediate aggressiveness between that of benign (WHO grade I) and anaplastic (WHO grade III) variants. ${ }^{8,23}$ Historically, maximal safe resection has been the primary treatment for atypical meningioma, but even

ABBREVIATIONS EBRT = external beam radiotherapy; EORTC = European Organization for Research and Treatment of Cancer; GTR = gross-total resection; IQR = interquartile range; KPS = Karnofsky Performance Scale; LFFR = local freedom from recurrence; LI = labeling index; MVA = multivariate analysis; OS = overall survival; RPA = recursive partitioning analysis; RT = radiotherapy; RTOG = Radiation Therapy Oncology Group; SRS = stereotactic radiosurgery; STR = subtotal resection.

SUBMITTED July 3, 2017. ACCEPTED September 5, 2017.

INCLUDE WHEN CITING Published online April 6, 2018; DOI: 10.3171/2017.9.JNS171609. 
after optimal resection, patients with atypical meningioma are at substantial risk of recurrence. ${ }^{3}$ Thus, some patients with atypical meningioma are treated with adjuvant radiotherapy (RT), but the indications for adjuvant RT, particularly after gross-total resection (GTR), have not been well established. Indeed, no prospective data are available to answer this question, although 3 clinical trials of adjuvant RT for atypical meningioma are currently ongoing (NCT03180268; ClinicalTrials.gov). ${ }^{16,32}$ As a result, criteria to guide adjuvant RT after resection of atypical meningioma is limited to data from retrospective studies with small sample sizes and mixed results that are confounded by differences in institutional practices. One histopathological factor that has been shown to have prognostic significance is the MIB1 labeling index (LI), which is a widely used marker of cellular proliferation across multiple tumor types, ${ }^{27,40}$ including atypical meningioma. ${ }^{11,26,29}$

Multiple studies have independently reported high rates of local control in patients receiving adjuvant RT following GTR of meningioma. . $^{1,2,5,14,21,34}$ Nevertheless, there is significant variability in treatment of atypical meningioma because limited data exist to identify patients who are most likely to benefit from adjuvant RT. ${ }^{35}$ The purpose of this study was to investigate the impact of adjuvant RT on local recurrence and overall survival (OS) in patients undergoing primary resection of atypical meningioma, and to identify predictive factors to guide patient selection for adjuvant RT.

\section{Methods}

\section{Study Design and Patient Population}

We performed a retrospective chart review of patients who underwent primary resection of atypical meningioma at a single institution between 1993 and 2014. Follow-up was monitored through May 2017. Study subjects were identified from institutional databases capturing all patients who underwent surgery for meningioma. Clinical and histopathological variables were collected from the electronic medical record, pathology databases, and radiology archives. This study was approved by the Institutional Review Board, Human Research Protection Program Committee.

\section{Clinical and Histopathological Variables}

Clinical variables assessed included age at the time of surgery, sex, meningioma location, extent of resection, preoperative Karnofsky Performance Scale (KPS) score, history of prior cranial RT, and receipt of adjuvant RT. Meningioma grade was based on the WHO criteria at the time of surgery, and all meningiomas included in subsequent analyses were WHO grade II. Retrospective review was performed for 67 cases (37\%), and confirmed the diagnosis of atypical meningioma in all instances. Radiation modality, dose, and adverse events (Radiation Therapy Oncology Group [RTOG] grade $>1)^{39}$ were also collected from the medical record. Histopathological data collected from pathology archives included the following: 1) MIB1 $\mathrm{LI}$; 2) the number of mitoses per $10 \mathrm{hpf}$; and reports of 3) sheeting or loss of architecture, 4) increased cellularity, 5) foci of spontaneous necrosis, 6) nuclear pleomorphism or atypia, 7) brain invasion, and 8) bone involvement. Grosstotal resection (Simpson grade I-III) and subtotal resection (STR; Simpson grade > III) were defined radiographically based on postoperative imaging, in conjunction with Simpson grade as determined by the surgeon at the time of operation (52 patients, 29\%). All postoperative scans occurred within 48 hours of surgery. If not explicitly stated, the KPS score was retrospectively determined based on the preoperative consultation records.

\section{Recurrence and Survival}

Meningioma recurrence was defined radiographically. After GTR, this meant local recurrence of any size on subsequent brain imaging. After STR, RECIST (Response Evaluation Criteria in Solid Tumours) criteria $^{10}$ were adapted to define recurrence of residual meningioma as interval growth of $\geq 20 \%$ along any dimension. Survival time and freedom from recurrence were measured from the date of resection. In determining freedom from recurrence, patients were censored at the date of last imaging. Survival status of patients was collected by a combined search of the electronic medical record, institutional cancer registry, and the SEER (Surveillance, Epidemiology, and End Results), Department of Motor Vehicles, social security, and nationwide hospital obituary databases, as well as a search of publicly available obituaries.

\section{Statistical Analysis}

All statistical analyses were performed using JMP 13.0 (SAS). To identify covariates associated with local freedom from recurrence (LFFR) and OS, Kaplan-Meier analyses were performed on each categorical variable of interest, with $\mathrm{p}$ values calculated by log-rank tests. Continuous or ordinal variables, including patient age at the time of surgery, MIB1 LI, mitoses/hpf, and KPS score, were dichotomized by determining an optimal cutoff point via receiver operating characteristic curve analysis. Variables with a $p$ value $\leq 0.1$ by log-rank test were included in multivariate Cox proportional hazards modeling analyses: multivariate analysis (MVA) and recursive partitioning analysis (RPA). The MVA outputs are displayed as relative risk with 95\% confidence interval. The RPA was performed to further identify subsets of patients at greater risk of recurrence. The number of partitions chosen was based on maximal $\mathrm{R}^{2}$ as calculated by $\mathrm{k}$-fold cross-validation $(\mathrm{k}=5)$.

\section{Results}

\section{Patient Characteristics and Clinical Outcomes}

We identified 182 patients who underwent primary resection of atypical meningioma between 1993 and 2014 (Table 1). The median imaging follow-up was 4.4 years (interquartile range [IQR] 1.8-7.5 years), and the median survival status follow-up among all patients was 6.0 years (IQR 3.7-9.7 years). The median KPS score was 80 (IQR 60-80). A GTR was achieved in 114 cases $(63 \%)$ and 42 patients $(23 \%)$ received adjuvant RT. Adjuvant RT was more common after STR $(\mathrm{p}<0.0001)$ and for skull base meningiomas $(p=0.02)$. Otherwise, there were no differences in patient age, sex, preoperative KPS score, history 
TABLE 1. Patient and tumor characteristics in 182 cases of atypical meningioma

\begin{tabular}{lccc}
\hline \multicolumn{1}{c}{ Characteristic } & $\begin{array}{c}\mathrm{RT}, \\
\mathrm{n}=42\end{array}$ & $\begin{array}{c}\text { No RT, } \\
\mathrm{n}=140\end{array}$ & $\begin{array}{c}\mathrm{p} \\
\text { Value }\end{array}$ \\
\hline Age in yrs (average \pm SD) & $56.5 \pm 14.3$ & $56.5 \pm 15.7$ & 1.00 \\
\hline Female & $27(64 \%)$ & $84(60 \%)$ & 0.65 \\
\hline Tumor site & & & 0.02 \\
\hline Skull base & $23(55 \%)$ & $49(35 \%)$ & \\
\hline Non-skull base & $19(45 \%)$ & $91(65 \%)$ & \\
\hline GTR & $10(24 \%)$ & $103(74 \%)$ & $<0.0001$ \\
\hline KPS score $>70 *$ & $23(56 \%)$ & $69(52 \%)$ & 0.64 \\
\hline Histopathology ${ }^{*}$ & & & \\
\hline MIB1 LI (average \pm SD) & $9.4 \pm 7.4 \%$ & $7.7 \pm 5.6 \%$ & 0.17 \\
\hline Mitoses/hpf $\leq 5$ & $7(17 \%)$ & $22(17 \%)$ & 0.97 \\
\hline Sheeting/loss of architecture & $24(59 \%)$ & $64(50 \%)$ & 0.39 \\
\hline Increased cellularity & $20(49 \%)$ & $75(59 \%)$ & 0.23 \\
\hline Foci of spontaneous necrosis & $7(17 \%)$ & $27(21 \%)$ & 0.69 \\
\hline Nuclear pleomorphism/atypia & $12(29 \%)$ & $47(37 \%)$ & 0.35 \\
\hline Brain invasion & $10(24 \%)$ & $32(25 \%)$ & 0.90 \\
\hline Bone involvement & $8(19 \%)$ & $20(16 \%)$ & 0.52 \\
\hline Prior cranial RT & $3(7 \%)$ & $10(7 \%)$ & 0.99 \\
\hline 5-yr LFFR/OS & $82 \% / 85 \%$ & $65 \% / 87 \%$ & $0.08 / 0.64$ \\
\hline Bof & & & \\
\hline
\end{tabular}

Boldface type indicates statistical significance.

* Preoperative KPS score could not be accurately determined based on the medical record in $8(4 \%)$ patients.

† Pathology reports did not contain detailed annotation of histopathological features in $13(7 \%)$ patients, including 1 patient who received RT and 12 who did not.

of prior cranial RT, or histopathological features with and without adjuvant RT.

To account for changes in WHO grading criteria over the study period, we performed univariate analysis of demographic and tumor characteristics stratified by 3 time periods corresponding to WHO editions: 1993-2000, 2001-2007, and 2008-2014. There were no significant differences in patient age, sex, preoperative KPS score, skull base location, history of prior cranial RT, or extent of resection among the cases from these time periods, although there was a trend toward greater use of adjuvant RT over time $(11 \%, 18 \%$, and $30 \%$, respectively; $\mathrm{p}=0.06)$. In addition, there were no significant differences between MIB1 $\mathrm{LI}$, mitoses/hpf, presence of brain invasion, recurrence ( $\mathrm{p}$ $=0.13, \log$-rank test $)$ or survival $(\mathrm{p}=0.49$, log-rank test) among the cases from these time periods.

The predominant radiation modality was external beam RT (EBRT) (86\%, median dose 59.4 Gy, range 36-60 Gy); a minority of patients received adjuvant stereotactic radiosurgery (SRS) (14\%). One patient with a history of prior craniospinal radiation for medulloblastoma received a reduced EBRT dose of $36 \mathrm{~Gy}$. The median time to radiation treatment was 10.6 weeks, to allow for adequate postoperative healing. Grade 2+ adverse effects of RT occurred in 5 patients $(12 \%)$. Two patients $(5 \%)$ experienced radiation necrosis, 1 patient experienced progressive spastic lower-extremity paresis attributed to radiation, 1 patient experienced short-term visual blurring that improved with

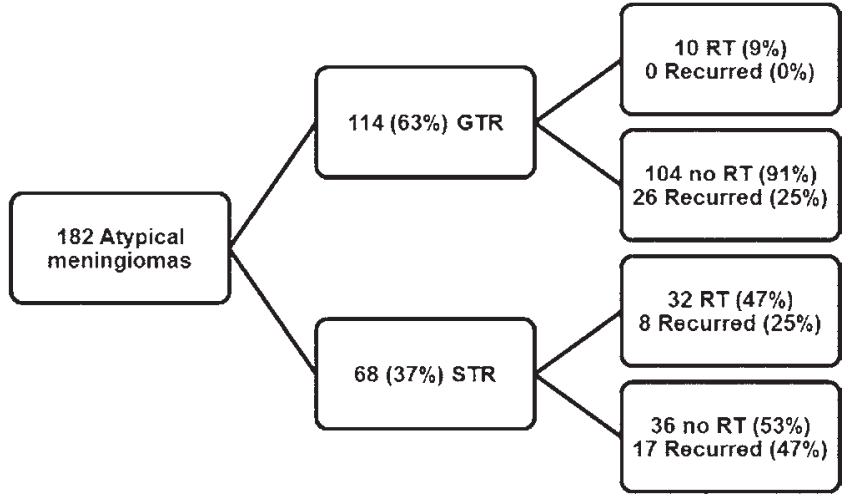

FIG. 1. Schematic showing study design.

steroids, and 1 patient experienced transient radiation-induced encephalopathy that responded to steroids. One patient $(3 \%)$ who experienced radiation necrosis developed intracranial hemorrhage following craniotomy for the necrosis, and died several months later.

The study design and Kaplan-Meier curves detailing clinical outcomes for patients based on extent of resection and adjuvant RT are shown in Figs. 1-3. During the study period, 51 patients experienced local recurrence (28\%) and 45 died (25\%). The LFFR at 5 years was $69 \%$ overall (95\% CI 61\%-77\%), $72 \%$ with GTR versus $64 \%$ with STR $(\mathrm{p}=0.03)$ (Fig. 2A), and $82 \%$ with RT versus $65 \%$ without RT $(\mathrm{p}=0.08)$ (Table 1$)$. The OS at 5 years was $86 \%$ overall (95\% CI 81\%-92\%); $91 \%$ with GTR versus $81 \%$ with STR $(p=0.02)$ (Fig. $2 B$ ); and $85 \%$ with RT versus $87 \%$ without $(\mathrm{p}=0.64)$. Cause of death was identified for 30 patients who died (67\%). After censoring patients with an unknown cause of death, the disease-specific survival at 5 years was $93 \%$ overall (95\% CI 89\%-97\%), 95\% with GTR versus $92 \%$ with STR ( $p=0.14, \log$-rank test), and $95 \%$ with RT versus $93 \%$ without ( $p=0.86, \log$-rank test).

Clinical and histopathological factors associated with LFFR and OS on Kaplan-Meier analysis are summarized in Table 2. Prognostic factors on MVA (Table 3) for local progression included GTR (RR 0.19, 95\% CI 0.06-0.54, $\mathrm{p}=0.0018)$; adjuvant RT (RR 0.15, 95\% CI 0.05-0.43, p $=0.0004) ; \mathrm{MIB} 1 \mathrm{LI} \leq 7 \%(\mathrm{RR} 0.21,95 \%$ CI $0.07-0.53, \mathrm{p}$ $=0.0009)$; and a remote history of prior cranial RT (RR
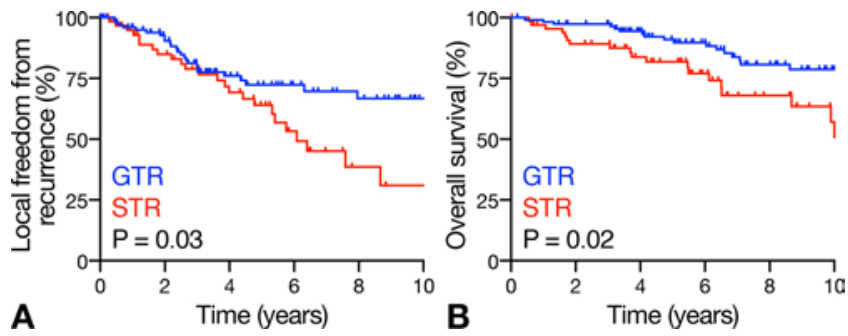

FIG. 2. Graphs showing outcomes of atypical meningioma according to extent of resection. A: The LFFR according to extent of resection. A GTR (blue) was associated with improved local control by log-rank test $(p=0.03)$ and MVA (RR 0.2, 95\% Cl 0.1-0.5, $p=0.002)$. B: The OS by extent of resection. A GTR (blue) was associated with improved OS by log-rank test $(p=0.02)$ and MVA (RR $0.4,95 \% \mathrm{Cl} 0.1-0.9, p=0.02)$. Figure is available in color online only. 

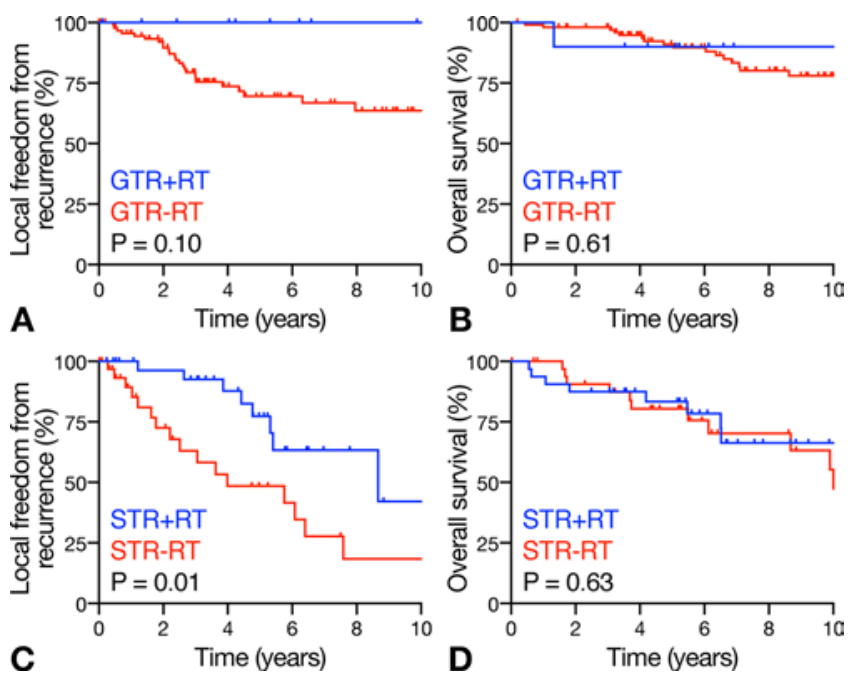

FIG. 3. Graphs showing outcomes of atypical meningioma according to adjuvant RT. A: The LFFR after GTR, stratified by adjuvant RT. There was a trend toward improved LFFR with GTR+RT by log-rank test ( $p$ $=0.10)$ that was significant on MVA $(p=0.01)$. B: The OS after GTR, stratified by adjuvant RT. There was no significant difference between groups ( $p=0.61$, log-rank test). C: The LFFR after STR, stratified by adjuvant RT. Adjuvant RT was associated with improved LFFR by logrank test $(p=0.01)$ and MVA (RR 0.2, 95\% Cl 0.04-0.7, $p=0.009)$.

D: The OS after STR, stratified by adjuvant RT. There was no significant difference between groups ( $p=0.63$, log-rank test). Figure is available in color online only.

$5.65,95 \%$ CI $1.25-18.78, \mathrm{p}=0.03)$. With respect to the latter, 5 patients had a remote history of prior cranial RT for childhood leukemia, 6 for childhood nonmeningioma brain tumors, 1 for adult optic glioma, and 1 for vestibu- lar schwannoma (Table 1). Prognostic factors on MVA for death from any cause included GTR (RR $0.35,95 \%$ CI $0.14-0.86, p=0.02$ ); MIB1 LI $\leq 7 \%$ (RR $0.38,95 \%$ CI $0.14-0.94, \mathrm{p}=0.04)$; and age at the time of surgery (RR 1.08 per year increase, $95 \%$ CI $1.05-1.12$, p < 0.0001). No other clinical or histopathological features reached statistical significance for LFFR or OS on multivariate analysis.

\section{Adjuvant RT After GTR}

There were 26 local recurrences after GTR (23\%), none of which received adjuvant RT. After GTR, an MIB1 LI $\leq 7 \%$ was associated with improved LFFR on MVA (RR $0.06,95 \%$ CI $0.003-0.32, p=0.0004)$. There was a trend toward improved LFFR with adjuvant RT on univariate Kaplan-Meier analysis $(\mathrm{p}=0.10)$ that reached statistical significance after adjusting for covariates on MVA ( $\mathrm{p}=$ 0.01 ). Indeed, there were no recurrences in the 10 patients treated with GTR and adjuvant RT (Fig. 3A). The mean MIB1 LI for the 10 meningiomas treated with GTR and adjuvant RT was $9.5 \% \pm 5.7 \%$ (range $3.6 \%-19.1 \%$ ), which was not significantly different from the meningiomas treated with GTR alone $(7.7 \% \pm 5.6 \%$, range $1.0 \%-30.0 \%)$. There was no significant difference in OS after GTR with or without adjuvant RT (Fig. 3B).

Recursive partitioning analysis was performed to identify variables associated with an elevated risk of local progression after GTR of atypical meningioma (Fig. 4). Consistent with MVA, the first partition was based on the MIB1 LI, with a cutoff of 7\%: $2.2 \%$ of meningiomas with MIB1 LI $\leq 7 \%$ recurred (1 of 45 ), as opposed to $36 \%$ of meningiomas with MIB1 LI > 7\% (25 of 69). Next, RPA partitioned meningiomas with MIB1 LI $>7 \%$ based on adjuvant RT, which again was consistent with MVA: none

TABLE 2. Univariate analysis of outcomes of atypical meningioma according to clinical and histopathological features

\begin{tabular}{|c|c|c|c|c|c|c|}
\hline \multirow[b]{2}{*}{ Feature } & \multicolumn{3}{|c|}{ LFFR } & \multicolumn{3}{|c|}{ OS } \\
\hline & GTR+STR & GTR & STR & GTR+STR & GTR & STR \\
\hline Age $>65$ yrs & 0.8 & 0.24 & 0.44 & $<0.0001$ & $<0.0001$ & 0.001 \\
\hline Female & 0.55 & 0.55 & 0.21 & 0.48 & 0.4 & 0.57 \\
\hline Skull base & 0.68 & 0.97 & $<0.05$ & 0.87 & 0.50 & 0.98 \\
\hline KPS score $>70$ & 0.17 & 0.77 & 0.06 & 0.13 & 0.66 & 0.12 \\
\hline GTR & 0.03 & - & - & 0.02 & - & - \\
\hline RT & 0.08 & 0.1 & 0.01 & 0.64 & 0.61 & 0.63 \\
\hline \multicolumn{7}{|l|}{ Histopathology } \\
\hline MIB1 LI $\leq 7 \%$ & $<0.0001$ & $<0.0001$ & 0.51 & 0.01 & 0.31 & 0.01 \\
\hline Mitoses/hpf $\leq 5$ & $<0.0001$ & 0.006 & 0.003 & 0.29 & 0.49 & 0.52 \\
\hline Sheeting/loss of architecture & 0.07 & 0.34 & 0.32 & 0.06 & 0.09 & 0.62 \\
\hline Increased cellularity & 0.7 & 0.19 & 0.06 & 0.29 & 0.38 & 0.7 \\
\hline Small cell change & 0.59 & 0.41 & 0.53 & 0.73 & 0.8 & 0.34 \\
\hline Foci of spontaneous necrosis & 0.42 & 0.72 & 0.21 & 0.78 & 0.25 & 0.7 \\
\hline Nuclear pleomorphism/atypia & 0.31 & 0.65 & 0.02 & 0.82 & 0.39 & 0.54 \\
\hline Brain invasion & 0.22 & 0.03 & 0.32 & 0.49 & 0.22 & 0.6 \\
\hline Bone involvement & 0.38 & 0.07 & 0.51 & 0.77 & 0.45 & 0.94 \\
\hline Prior cranial RT & 0.06 & 0.03 & 0.53 & 0.7 & 0.16 & 0.43 \\
\hline
\end{tabular}

The $p$ values were calculated by Kaplan-Meier analysis and log-rank tests. Boldface type indicates statistical significance. Covariates with $p$ values $\leq 0.10$ were included in subsequent MVAs and RPA. 
TABLE 3. Multivariate Cox analyses

\begin{tabular}{|c|c|c|c|}
\hline Variable & $\mathrm{RR}$ & $95 \% \mathrm{Cl}$ & $p$ Value \\
\hline \multicolumn{4}{|l|}{ Local recurrence, all patients } \\
\hline GTR & 0.19 & $0.06-0.54$ & 0.0018 \\
\hline Adjuvant RT & 0.15 & $0.05-0.43$ & 0.0004 \\
\hline MIB1 LI $\leq 7 \%$ & 0.21 & $0.07-0.53$ & 0.0009 \\
\hline Mitoses/hpf $\leq 5$ & 0.62 & $0.25-1.69$ & 0.34 \\
\hline Sheeting/loss of architecture & 1.09 & $0.44-2.73$ & 0.86 \\
\hline Prior cranial RT & 5.65 & $1.25-18.78$ & 0.03 \\
\hline \multicolumn{4}{|l|}{ Local recurrence, tumors w/ GTR } \\
\hline Adjuvant RT & 0 & $0-0.46$ & 0.01 \\
\hline MIB1 LI $\leq 7 \%$ & 0.06 & $0.003-0.32$ & 0.0004 \\
\hline Mitoses/hpf $\leq 5$ & 0.4 & $0.10-1.69$ & 0.21 \\
\hline Brain invasion & 1.54 & $0.44-5.49$ & 0.5 \\
\hline Bone invasion & 4.07 & $0.79-16.71$ & 0.09 \\
\hline Prior cranial RT & 2.79 & $0.33-15.85$ & 0.3 \\
\hline \multicolumn{4}{|l|}{ Local recurrence, tumors w/ STR } \\
\hline Skull base & 0.28 & $0.07-1.04$ & 0.06 \\
\hline KPS score $>70$ & 0.53 & $0.18-1.55$ & 0.24 \\
\hline Adjuvant RT & 0.18 & $0.04-0.66$ & 0.0089 \\
\hline Mitoses/hpf $\leq 5$ & 0.12 & $0.03-0.44$ & 0.0018 \\
\hline Increased cellularity & 1.74 & $0.45-7.34$ & 0.43 \\
\hline Nuclear pleomorphism & 1.75 & $0.50-6.18$ & 0.38 \\
\hline \multicolumn{4}{|l|}{ OS, all patients } \\
\hline Age & $\begin{array}{c}\text { 1.08/yr } \\
\text { increase }\end{array}$ & $1.05-1.12$ & $<0.0001$ \\
\hline GTR & 0.35 & $0.14-0.86$ & 0.02 \\
\hline MIB1 LI $\leq 7 \%$ & 0.38 & $0.14-0.94$ & 0.04 \\
\hline Sheeting/loss of architecture & 1.35 & $0.56-3.46$ & 0.51 \\
\hline
\end{tabular}

Boldface type indicates statistical significance.

of the tumors with MIB1 LI $>7 \%$ treated with GTR and adjuvant RT recurred ( 0 of 6 ), as opposed to $40 \%$ of tumors with MIB1 LI > 7\% treated with GTR alone (25 of 63). Finally, RPA partitioned meningiomas with MIB1 LI $>7 \%$ that did not receive adjuvant RT based on brain or bone invasion: $33 \%$ of tumors with MIB1 LI $>7 \%$ but no brain or bone invasion treated with GTR alone recurred (13 of 40), as opposed to $52 \%$ of tumors with MIB1 LI >
$7 \%$ and brain or bone invasion treated with GTR alone (12 of 23).

\section{Adjuvant RT After STR}

Sixty-eight patients underwent STR (37\%), after which 32 received adjuvant RT (47\%) and 25 tumors recurred (37\%) (Table 1). Twenty-five percent of patients who received RT experienced recurrence (8 of 32) compared with $47 \%$ of those who did not receive RT (17 of 36). After adjusting for covariates on MVA, adjuvant RT (RR 0.18, 95\% CI 0.04-0.66, $\mathrm{p}=0.009$ ) (Fig. 3C) and $\leq 5$ mitoses per $10 \mathrm{hpf}$ (RR 0.12, 95\% CI 0.03-0.44, $\mathrm{p}=0.0018$ ) were predictive for local progression. There was no significant difference in OS after STR with or without adjuvant RT (Fig. 3D), and RPA failed to identify any robust variables associated with an elevated risk of local progression after STR of atypical meningioma.

\section{Salvage Treatment at Recurrence}

Of 51 patients in whom lesions recurred, $8(16 \%)$ underwent repeat surgery (5 with adjuvant RT), 20 (39\%) underwent SRS, $10(20 \%)$ underwent EBRT, 1 (2\%) was observed, and $12(23 \%)$ were lost to follow-up. Tumors in 17 of $38(45 \%)$ patients who underwent salvage treatment subsequently re-recurred, and the median time to rerecurrence was 3.1 years. Kaplan-Meier analysis did not reveal a significant difference in LFFR between patients treated with EBRT versus SRS at recurrence $(\mathrm{p}=0.95$, log-rank test), although there was a significant difference between patients treated at recurrence with RT versus surgery $(\mathrm{p}=0.0006, \log$-rank test). In that regard, patients treated with surgery at recurrence were more likely to experience tumor re-recurrence ( 7 of 8 patients, $88 \%$ ), with a median time to re-recurrence of 1.1 years.

\section{Discussion \\ Key Findings}

Adjuvant RT improved LFFR of atypical meningioma irrespective of the extent of resection in this single-institution cohort with intermediate to long-term follow-up. An MIB1 LI $\leq 7 \%$ was a strong predictive factor for longer LFFR and longer OS after GTR. Consistently, RPA identified a subset of atypical meningiomas with MIB1 LI > 7\% treated with GTR that appeared to benefit most from ad-

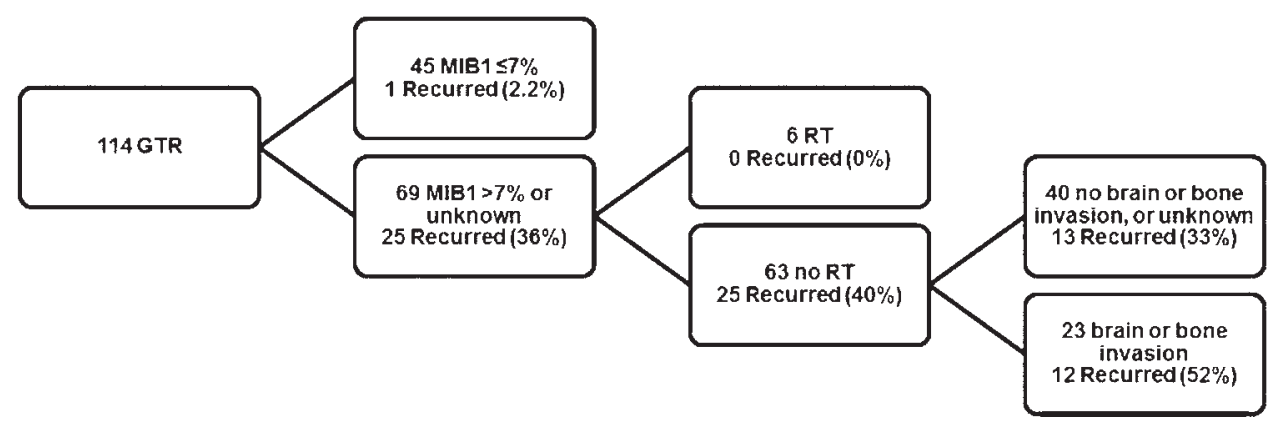

FIG. 4. Schematic showing RPA for atypical meningioma status post-GTR. Optimal number of splits (3) was based on $\mathrm{R}^{2}$ as calculated by $k$-fold cross-validation $(k=5)$. 
juvant RT. A count of $\leq 5$ mitoses per 10 hpf was a strong predictive factor for decreased local recurrence after STR.

Our study was unable to identify an OS benefit with adjuvant RT for atypical meningioma. This result should be interpreted in light of the fact that there are many effective salvage treatments for meningioma even after adjuvant RT, including radiosurgery, repeat resection, and repeat resection with adjuvant brachytherapy. ${ }^{2,19,24,31,38}$ Another possibility is that the follow-up in our study was too brief for a benefit in disease-specific survival to produce a significant benefit in OS. Ultimately, adequately powered prospective trials with survival parameters as long-term end points will be needed to satisfactorily elucidate the effect of adjuvant RT on OS from atypical meningioma.

\section{Adjuvant RT After GTR of Atypical Meningioma}

The literature arguing for or against adjuvant RT after GTR of atypical meningioma is controversial. Studies have shown questionable or no benefit, ${ }^{8,12,14,15,20,25,29,37}$ a trend toward benefit, ${ }^{1,11,13,17}$ or significant benefit ${ }^{2,5,29,30,34}$ with adjuvant RT. Multiple studies have independently reported $100 \% 1,14,34$ or near- $100 \% \%^{2,5,21}$ local control after GTR and adjuvant RT, albeit with small sample sizes of 8-22 patients. These encouraging data appear to be validated by the initial 3-year results from RTOG $0539,{ }^{32}$ a Phase II nonrandomized trial that showed 3-year progression-free survival of $96 \%$ in 52 intermediate-risk meningiomas that received adjuvant RT, including 36 atypical meningiomas treated with GTR and 16 recurrent WHO grade I meningiomas. Another ongoing trial, the European Organization for Research and Treatment of Cancer (EORTC) 1308, is a randomized controlled trial of adjuvant RT after GTR of atypical meningioma, although the outcome data are not yet available. ${ }^{16}$ Finally, a Phase III trial randomizing patients to observation versus adjuvant RT after GTR, NRG-BN003, was opened in June 2017. Thus, our findings corroborate and add to the growing body of evidence supporting adjuvant RT after GTR of atypical meningioma, and provide a foundation for molecular analyses of the prospective tissue assembled for the aforementioned trials.

\section{Risk Factors for Recurrence After GTR of Atypical Meningiomas}

Should the long-term results of RTOG 0539, EORTC 1308, and NRG-BN003 demonstrate a benefit of adjuvant RT for atypical meningioma, it will become increasingly important to identify predictive factors for recurrence after GTR to appropriately select patients for adjuvant RT and spare those at very low risk of recurrence from the potential adverse effects of treatment. This need is highlighted by the occurrence of several serious toxicities in our patients who received adjuvant RT.

Prior studies have suggested that brain invasion, ${ }^{26,42}$ bone involvement, ${ }^{12}$ higher Simpson grade, ${ }^{18}$ and elevated MIB1 LI ${ }^{11,29,41}$ are associated with increased risk of recurrence among atypical meningiomas. Our findings support the use of MIB1 in stratifying patients for adjuvant RT after GTR. The MIB1 LI has the advantage of being a well-known, semiquantitative marker of cellular proliferation with proven prognostic significance across multiple tumor types, ${ }^{27,40}$ including atypical meningioma. ${ }^{6,11,26,29,41}$ After GTR, atypical meningiomas with MIB1 LI $\leq 7 \%$ had a very low risk of recurrence, suggesting that patients who meet these criteria may benefit less from adjuvant RT. Conversely, brain invasion was associated with increased recurrence, consistent with prior studies. ${ }^{26,42}$ Thus, after GTR, atypical meningiomas with a high MIB1 LI and brain or bone invasion may benefit most from adjuvant RT. Future studies identifying and validating risk factors for recurrence in atypical meningioma, as well as expanding on recently identified molecular and methylation-based markers of meningioma subtypes are warranted.,33

\section{Strengths and Limitations}

The results of this study should be interpreted in the context of several strengths and limitations. With respect to strengths, our study included a relatively large number of patients with nearly 5 years of imaging follow-up, allowing for multivariate Cox modeling and investigation of multiple histopathological features in relation to LFFR and OS.

With respect to limitations, the retrospective nature of our study introduces the potential for selection bias among patients who received adjuvant RT. Patients with and without adjuvant RT did not differ in age, sex, KPS score, remote history of prior cranial RT, or in meningioma histopathology, including MIB1 LI. However, patients who received adjuvant RT were less likely to have undergone GTR. We accounted for this discrepancy by analyzing adjuvant RT after GTR and STR separately. Patients who received adjuvant RT were also less likely to have convexity meningioma. This finding was most likely due to the higher rate of GTR of convexity meningioma, which may be a product of comparative ease of access by the surgeon. Nevertheless, the significance of the difference in meningioma location to our findings is not clear. In addition, our finding of a benefit in LFFR with adjuvant RT after GTR, although concordant with other recent reports, should be interpreted in the context of our very small sample size of 10 patients.

Our finding that the small subset of patients who underwent repeat surgery at recurrence had a high rate of rerecurrence should be interpreted with caution, because it most likely represents a selection bias toward aggressively recurrent tumors, which were not amenable to radiosurgery or EBRT. Further studies examining outcomes among different salvage treatment modalities are warranted.

Determination of extent of resection in our study was based on postoperative imaging in conjunction with Simpson grade as reported by the surgeon. Although this definition of GTR mirrors clinical practice at our institution, questions remain with regard to the potential impact of the timing of postoperative scans, differences in institutional practices, and experience with the determination of resection extent, all of which may limit the generalizability of our results.

Another possible limitation of this study relates to the changes in WHO grading criteria for atypical meningioma in 2000 and $2007,,^{20,22}$ which have had the reported effect of increasing the proportion of atypical versus benign meningiomas diagnosed, ${ }^{4,36}$ potentially changing the 
characteristics of tumors diagnosed in different eras. We attempted to account for these changes in grading criteria by stratifying by WHO era, and found no significant differences in patient demographics or tumor characteristics, although there was a trend toward greater use of adjuvant RT over time.

Finally, the various cutoffs identified in our study should be interpreted with caution. For instance, it is well recognized that interlaboratory variations of technique and interpretation make it difficult to establish MIB1 LI cutoffs that can be universally applied; as such, each laboratory must determine its own cutoff for an elevated LI locally.

\section{Conclusions}

Adjuvant RT reduces local progression of atypical meningioma irrespective of extent of resection, and is well tolerated. Although independent validation is required, our results suggest that the MIB1 LI, the number of mitoses per $10 \mathrm{hpf}$, and brain or bone invasion may be useful guides to the selection of patients who are most likely to benefit from adjuvant RT after resection of atypical meningioma.

\section{Acknowledgments}

This work was supported by grants from the Linda Wolfe Meningioma Research Fund and the UCSF Physician Scientist Scholar Program to D.R.R., and from the NIH (1F32CA21394401) to S.T.M.

\section{References}

1. Aghi MK, Carter BS, Cosgrove GR, Ojemann RG, AminHanjani S, Martuza RL, et al: Long-term recurrence rates of atypical meningiomas after gross total resection with or without postoperative adjuvant radiation. Neurosurgery 64:56-60, 2009

2. Aizer AA, Arvold ND, Catalano P, Claus EB, Golby AJ, Johnson MD, et al: Adjuvant radiation therapy, local recurrence, and the need for salvage therapy in atypical meningioma. Neuro Oncol 16:1547-1553, 2014

3. Aizer AA, Bi WL, Kandola MS, Lee EQ, Nayak L, Rinne ML, et al: Extent of resection and overall survival for patients with atypical and malignant meningioma. Cancer 121:43764381, 2015

4. Backer-Grøndahl T, Moen BH, Torp SH: The histopathological spectrum of human meningiomas. Int J Clin Exp Pathol 5:231-242, 2012

5. Bagshaw HP, Burt LM, Jensen RL, Suneja G, Palmer CA, Couldwell WT, et al: Adjuvant radiotherapy for atypical meningiomas. J Neurosurg 126:1822-1828, 2017

6. Bruna J, Brell M, Ferrer I, Gimenez-Bonafe P, Tortosa A: Ki67 proliferative index predicts clinical outcome in patients with atypical or anaplastic meningioma. Neuropathology 27:114-120, 2007

7. Clark VE, Erson-Omay EZ, Serin A, Yin J, Cotney J, Ozduman K, et al: Genomic analysis of non-NF2 meningiomas reveals mutations in TRAF7, KLF4, AKT1, and SMO. Science 339: 1077-1080, 2013

8. Commins DL, Atkinson RD, Burnett ME: Review of meningioma histopathology. Neurosurg Focus 23(4):E3, 2007

9. Davis FG, Kupelian V, Freels S, McCarthy B, Surawicz T: Prevalence estimates for primary brain tumors in the United States by behavior and major histology groups. Neuro Oncol 3:152-158, 2001

10. Eisenhauer EA, Therasse P, Bogaerts J, Schwartz LH, Sar- gent $\mathrm{D}$, Ford R, et al: New response evaluation criteria in solid tumours: revised RECIST guideline (version 1.1). Eur J Cancer 45:228-247, 2009

11. Endo T, Narisawa A, Ali HSM, Murakami K, Watanabe T, Watanabe M, et al: A study of prognostic factors in 45 cases of atypical meningioma. Acta Neurochir (Wien) 158:16611667,2016

12. Gabeau-Lacet D, Aghi M, Betensky RA, Barker FG, Loeffler JS, Louis DN: Bone involvement predicts poor outcome in atypical meningioma. J Neurosurg 111:464-471, 2009

13. Hammouche S, Clark S, Wong AHL, Eldridge P, Farah JO: Long-term survival analysis of atypical meningiomas: survival rates, prognostic factors, operative and radiotherapy treatment. Acta Neurochir (Wien) 156:1475-1481, 2014

14. Hardesty DA, Wolf AB, Brachman DG, McBride HL, Youssef E, Nakaji P, et al: The impact of adjuvant stereotactic radiosurgery on atypical meningioma recurrence following aggressive microsurgical resection. J Neurosurg 119:475481,2013

15. Ildan F, Erman T, Göçer AI, Tuna M, Bağdatoğlu H, Çetinalp E, et al: Predicting the probability of meningioma recurrence in the preoperative and early postoperative period: a multivariate analysis in the midterm follow-up. Skull Base 17:157-171, 2007

16. Jenkinson MD, Javadpour M, Haylock BJ, Young B, Gillard H, Vinten J, et al: The ROAM/EORTC-1308 trial: Radiation versus Observation following surgical resection of Atypical Meningioma: study protocol for a randomised controlled trial. Trials 16:519, 2015

17. Jenkinson MD, Waqar M, Farah JO, Farrell M, Barbagallo GMV, McManus R, et al: Early adjuvant radiotherapy in the treatment of atypical meningioma. J Clin Neurosci 28:8792, 2016

18. Jo K, Park HJ, Nam DH, Lee JI, Kong DS, Park K, et al: Treatment of atypical meningioma. J Clin Neurosci 17:1362-1366, 2010

19. Kaprealian T, Raleigh DR, Sneed PK, Nabavizadeh N, Nakamura JL, McDermott MW: Parameters influencing local control of meningiomas treated with radiosurgery. J Neurooncol 128:357-364, 2016

20. Kleihues P, Louis DN, Scheithauer BW, Rorke LB, Reifenberger G, Burger PC, et al: The WHO classification of tumors of the nervous system. J Neuropathol Exp Neurol 61:215229,2002

21. Komotar RJ, Iorgulescu JB, Raper DMS, Holland EC, Beal $\mathrm{K}$, Bilsky MH, et al: The role of radiotherapy following gross-total resection of atypical meningiomas. J Neurosurg 117:679-686, 2012

22. Louis DN, Ohgaki H, Wiestler OD, Cavenee WK, Burger PC, Jouvet A, et al: The 2007 WHO classification of tumours of the central nervous system. Acta Neuropathol 114:97-109, 2007

23. Louis DN, Perry A, Reifenberger G, von Deimling A, Figarella-Branger D, Cavenee WK, et al: The 2016 World Health Organization Classification of Tumors of the Central Nervous System: a summary. Acta Neuropathol 131:803-820, 2016

24. Magill ST, Lau D, Raleigh DR, Sneed PK, Fogh SE, McDermott MW: Surgical resection and interstitial iodine-125 brachytherapy for high-grade meningiomas: a 25-year series. Neurosurgery 80:409-416, 2017

25. Mair R, Morris K, Scott I, Carroll TA: Radiotherapy for atypical meningiomas. J Neurosurg 115:811-819, 2011

26. Modha A, Gutin PH: Diagnosis and treatment of atypical and anaplastic meningiomas: a review. Neurosurgery 57:538550,2005

27. Moskowitz SI, Jin T, Prayson RA: Role of MIB1 in predicting survival in patients with glioblastomas. J Neurooncol 76:193-200, 2006

28. Ostrom QT, Gittleman H, Xu J, Kromer C, Wolinsky Y, 
Kruchko C, et al: CBTRUS statistical report: primary brain and other central nervous system tumors diagnosed in the United States in 2009-2013. Neuro Oncol 18 (Suppl 5):v1v75, 2016

29. Park H, Kim I, Jung H: Atypical meningioma: outcomes and prognostic factors. Int J Radiat Oncol Biol Phys 75 (3 Suppl):S238, 2009 (Abstract)

30. Pisćević I, Villa A, Milićević M, Ilić R, Nikitović M, Cavallo LM, et al: The influence of adjuvant radiotherapy in atypical and anaplastic meningiomas: a series of 88 patients in a single institution. World Neurosurg 83:987-995, 2015

31. Pourel N, Auque J, Bracard S, Hoffstetter S, Luporsi E, Vignaud JM, et al: Efficacy of external fractionated radiation therapy in the treatment of meningiomas: a 20-year experience. Radiother Oncol 61:65-70, 2001

32. Rogers L, Zhang P, Vogelbaum MA, Perry A, Ashby L, Modi $\mathrm{J}$, et al: Intermediate-risk meningioma: initial outcomes from NRG Oncology/RTOG-0539. Int J Radiat Oncol Biol Phys 93 (3 Suppl):S139-S140, 2015 (Abstract)

33. Sahm F, Schrimpf D, Stichel D, Jones DTW, Hielscher T, Schefzyk S, et al: DNA methylation-based classification and grading system for meningioma: a multicentre, retrospective analysis. Lancet Oncol 18:682-694, 2017

34. Shakir SI, Souhami L, Petrecca K, Mansure JJ, Khushdeep S, Panet-Raymond V, et al: Analysis of prognostic factors for local recurrence in atypical meningiomas. Int $\mathbf{J}$ Radiat Oncol Biol Phys 96 (2 Suppl):E78, 2016 (Abstract)

35. Simon M, Boström J, Koch P, Schramm J: Interinstitutional variance of postoperative radiotherapy and follow up for meningiomas in Germany: impact of changes of the WHO classification. J Neurol Neurosurg Psychiatry 77:767-773, 2006

36. Smith SJ, Boddu S, Macarthur DC: Atypical meningiomas: WHO moved the goalposts? Br J Neurosurg 21:588-592, 2007

37. Stessin AM, Schwartz A, Judanin G, Pannullo SC, Boockvar JA, Schwartz TH, et al: Does adjuvant external-beam radiotherapy improve outcomes for nonbenign meningiomas? A Surveillance, Epidemiology, and End Results (SEER)-based analysis. J Neurosurg 117:669-675, 2012

38. Sun SQ, Cai C, Murphy RKJ, DeWees T, Dacey RG, Grubb $\mathrm{RL}$, et al: Radiation therapy for residual or recurrent atypical meningioma: the effects of modality, timing, and tumor pathology on long-term outcomes. Neurosurgery 79:23-32, 2016
39. Trotti A, Byhardt R, Stetz J, Gwede C, Corn B, Fu K, et al: Common toxicity criteria: version 2.0. An improved reference for grading the acute effects of cancer treatment: impact on radiotherapy. Int J Radiat Oncol Biol Phys 47:13-47, 2000

40. Verhoven B, Yan Y, Ritter M, Khor LY, Hammond E, Jones $\mathrm{C}$, et al: Ki-67 is an independent predictor of metastasis and cause-specific mortality for prostate cancer patients treated on Radiation Therapy Oncology Group (RTOG) 94-08. Int J Radiat Oncol Biol Phys 86:317-323, 2013

41. Wang YC, Chuang CC, Wei KC, Chang CN, Lee ST, Wu CT, et al: Long term surgical outcome and prognostic factors of atypical and malignant meningiomas. Sci Rep 6:35743, 2016

42. Yang SY, Park CK, Park SH, Kim DG, Chung YS, Jung HW: Atypical and anaplastic meningiomas: prognostic implications of clinicopathological features. J Neurol Neurosurg Psychiatry 79:574-580, 2008

\section{Disclosures}

The authors report no conflict of interest concerning the materials or methods used in this study or the findings specified in this paper.

\section{Author Contributions}

Conception and design: Raleigh, Braunstein. Acquisition of data: Raleigh, Chen, Magill, Wu, Braunstein. Analysis and interpretation of data: Raleigh, Chen, Magill, Vasudevan, Morin, Braunstein. Drafting the article: Raleigh, Chen, Vasudevan, Morin, Aghi, Theodosopoulos, Perry, McDermott, Sneed, Braunstein. Critically revising the article: Raleigh, Chen, Magill, Vasudevan, Morin, Aghi, Theodosopoulos, Perry, McDermott, Sneed, Braunstein. Reviewed submitted version of manuscript: all authors. Approved the final version of the manuscript on behalf of all authors: Raleigh. Statistical analysis: Raleigh, Chen, Braunstein. Administrative/technical/material support: Raleigh, Chen. Study supervision: Raleigh, Braunstein.

\section{Correspondence}

David R. Raleigh: University of California, San Francisco, CA. david.raleigh@ucsf.edu. 